\title{
On the Gaussian Integration of Chebyshev Polynomials
}

\author{
By A. R. Curtis and P. Rabinowitz
}

\begin{abstract}
It is shown that as $m$ tends to infinity, the error in the integration of the Chebyshev polynomial of the first kind, $T_{(4 m+2) j_{ \pm 2} l}(x)$, by an $m$-point Gauss integration rule approaches $(-1)^{j} \cdot 2 /\left(4 l^{2}-1\right), l=0,1, \cdots, m-1$, and $(-1)^{j} \cdot \pi / 2, l=m$, for all $j$.
\end{abstract}

1. Knowledge of the errors in the numerical integration of Chebyshev polynomials of the first kind, $T_{n}(x)$, by given integration rules has proved to be useful in various situations. On the one hand, they can be used in estimating the error in integrating functions of low-order continuity [4] or with branch-point singularities [5]. On the other hand, they are needed in computing the norm of the error functional of the given rule in a certain family of Hilbert spaces of analytic functions [7]. For certain rules, namely for Gauss, Lobatto and Radau rules, asymptotic, and in some cases exact, values of these errors for certain values of the parameters involved were given in [3]. In this paper, we give further asymptotic results for the case of Gauss integration which are valid for all values of the parameters, thus completing the picture in this particular but very important case. These asymptotic results, which agree with the true computed results quite early, have been used to explain why the use of a Gauss rule with an even number of points, say $2 n$, is superior to one with an odd number of points, $2 n+1$, in integrating a function which is not analytic at the midpoint of the integration interval [6]. Numerical evidence suggests that similar results hold for a second family of important rules, the Lobatto rules, but the tools at our disposal in this case are not as powerful as in the well-investigated Gauss case and, hence, we were unable to prove these results.

2. The Chebyshev polynomials of the first kind, $T_{n}(x)$, are defined in $I=[-1,1]$ by $T_{n}(x)=\cos n \theta$, where $x=\cos \theta, 0 \leqq \theta \leqq \pi$. We shall denote the error in integrating $T_{n}(x)$, using a Gauss $m$-point rule, by

$$
E_{m}\left(T_{n}\right)=\int_{-1}^{1} T_{n}(x) d x-\sum_{i=1}^{m} w_{i} T_{n}\left(x_{i}\right)
$$

where $x_{i}=x_{i, m}, i=1, \cdots, m$, are the zeros of the Legendre polynomial of degree $m$ and $w_{i}=w_{i, m}, i=1, \cdots, m$, are the corresponding weights which are all positive with $\sum_{i=1}^{m} w_{i}=2$. Since $T_{n}(x)$ is odd for $n$ odd and since the Gauss rules are symmetric about the origin, it follows that $E_{m}\left(T_{2 k+1}\right)=0$ for all $k$. Furthermore, an $m$-point Gauss rule is exact for all polynomials of degree $\leqq 2 m-1$. Hence, we shall only consider $E_{m}\left(T_{2 k}\right)$, where $k \geqq m$. In this case, we have

Received March 12, 1971, revised June 6, 1971.

AMS 1970 subject classifications. Primary 65D30; Secondary 33A65.

Key words and phrases. Gaussian integration, Chebyshev polynomials of the first kind, asymptotic error, numerical integration error. 


$$
E_{m}\left(T_{2 k}\right)=\frac{2}{1-4 k^{2}}-\sum_{i=1}^{m} w_{i} T_{2 k}\left(x_{i}\right)
$$

For $k=m+j$, it has been shown in [3] that, as $m \rightarrow \infty$,

$$
\begin{aligned}
E_{m}\left(T_{2 m+2 j}\right) & =\pi / 2+O\left(m^{-1}\right), & & j=0, \\
& =-\pi / 2+O\left(m^{-1}\right), & & j=1, \\
& =O\left(m^{-1}\right), & & j>1,
\end{aligned}
$$

and, in fact, exact expressions have been given there for $E_{m}\left(T_{2 m+2 i}\right), j=0,1,2$, and it has been indicated how to obtain exact expressions for larger values of $j$. In Section 3 , we shall derive an asymptotic expression for $E_{m}\left(T_{2 k}\right)$ for the case $k=2 m+1$ which generalizes easily to $k=(2 m+1) j, j=2,3, \cdots$. In Section 4 , we do the same for the case $k=2 m+1 \pm l, l=1, \cdots, m$. This again generalizes to $k=(2 m+1) j \pm l$, $j=2,3, \cdots, l=1, \cdots, m$. Hence, we shall have obtained the asymptotic behavior of $E_{m}\left(T_{2 k}\right)$ for all $k$.

3. In the Gauss $m$-point rule, the abscissas $x_{i}, i=1, \cdots, m$, have the following asymptotic behavior [1, p. 787]:

Let $x_{i}=\cos \theta_{i}$, then

$$
\begin{aligned}
\theta_{i}=\theta_{i, m} & =\frac{4 i-1}{4 m+2} \pi+\cot \left(\frac{4 i-1}{4 m+2} \pi\right) / 8 m^{2}+O\left(m^{-8}\right) \\
& =\phi_{i}+\cot \phi_{i} / 8 m^{2}+O\left(m^{-8}\right),
\end{aligned}
$$

where $\phi_{i}=\phi_{i, m}=\pi(4 i-1) /(4 m+2)$.

It follows that

$$
\begin{aligned}
T_{4 m+2}\left(x_{i}\right) & =\cos \left((4 m+2) \theta_{i}\right) \\
& =\cos \left((4 i-1) \pi+\frac{4 m+2}{8 m^{2}} \cot \phi_{i}+O\left(m^{-2}\right)\right) \\
& =\cos \left((4 i-1) \pi+\frac{1}{2 m} \cot \phi_{i}+O\left(m^{-1}\right)\right) \\
& =\cos \left((4 i-1) \pi+h_{i}\right),
\end{aligned}
$$

where $h_{i}=(1 / 2 m) \cot \phi_{i}+O\left(m^{-1}\right)$. By Taylor's theorem,

$$
\cos \left((4 i-1) \pi+h_{i}\right)=-1-\frac{h_{i}^{2}}{2} \cos \left((4 i-1) \pi+t_{i} h_{i}\right)
$$

with $0<t_{i}<1$. Hence,

$$
\begin{aligned}
\sum_{i=1}^{m} w_{i} T_{4 m+2}\left(x_{i}\right) & =-\sum_{i=1}^{m} w_{i}-\sum_{i=1}^{m} w_{i} \cos \left((4 i-1) \pi+t_{i} h_{i}\right) \frac{h_{i}^{2}}{2} \\
& =-2-\sum_{i=1}^{m} w_{i} \cos \left((4 i-1) \pi+t_{i} h_{i}\right) \frac{h_{i}^{2}}{2}
\end{aligned}
$$

so that 


$$
\left|\sum_{i=1}^{m} w_{i} T_{4 m+2}\left(x_{i}\right)+2\right| \leqq \sum_{i=1}^{m} w_{i} h_{i}^{2} / 2 .
$$

We now show that $\sum_{i=1}^{m} w_{i} h_{i}^{2}=O\left(m^{-1}\right)$ which will imply that

$$
\begin{aligned}
E_{m}\left(T_{4 m+2}\right) & =\int_{-1}^{1} T_{4 m+2}(x) d x-\sum_{i=1}^{m} w_{i} T_{4 m+2}\left(x_{i}\right) \\
& =\frac{2}{1-(4 m+2)^{2}}+2+O\left(m^{-1}\right)=2+O\left(m^{-1}\right) .
\end{aligned}
$$

Since $h_{i}^{2}=\cot ^{2} \phi_{i} / 4 m^{2}+O\left(m^{-1}\right)$, it suffices to show that $\sum_{i=1}^{m} w_{i} \cot ^{2} \phi_{i}=O(m)$. This we do by showing that

$$
\begin{aligned}
\sum_{i=1}^{I(m+1) / 2]} w_{i} \cot ^{2} \phi_{i} & <K \int_{\pi /(4 m+2)}^{\pi / 2} \cot ^{2} \phi d \phi \\
& =\left.(-\cot \phi-\phi)\right|_{\pi /(4 m+2)} ^{\pi / 2}=O(m),
\end{aligned}
$$

since, by symmetry,

$$
2 \sum_{i=1}^{\mid(m+1) / 2\rfloor} w_{i} \cot ^{2} \phi_{i} \geqq \sum_{i=1}^{m} w_{i} \cot ^{2} \phi_{i} .
$$

Now, by [8, p. 351], $w_{i}=O\left(m^{-1}\right)$ uniformly in $i, i=1, \cdots,[(m+1) / 2]$, so that $w_{i}<K\left(\phi_{i}-\phi_{i-1}\right), i=1, \cdots,[(m+1) / 2]$, where $\phi_{0}=\pi /(4 m+2)$. Since $\cot ^{2} \phi$ is monotonically decreasing in $(0, \pi / 2]$, we have that $w_{i} \cot ^{2} \phi_{i}<K\left(\phi_{i}-\phi_{i-1}\right) \cot ^{2} \phi_{i}<K \int_{\phi_{i-1}}^{\phi_{i}} \cot ^{2} \phi d \phi, \quad i=1, \cdots,[(m+1) / 2]$, which proves our result.

Similar reasoning leads us to conclude that, for any fixed positive integer $j$,

$$
E\left(T_{(4 m+2) i}\right)=(-1)^{i+1} \cdot 2+O\left(m^{-1}\right) .
$$

4. Let us now consider

$$
T_{4 m+2 \pm 2 l}\left(x_{i}\right), \quad l=1, \cdots, m .
$$

This is equal to

$$
\begin{aligned}
\cos \left((4 m+2 \pm 2 l) \theta_{i}\right)= & \cos \left((4 m+2) \theta_{i}\right) \cos 2 l \theta_{i} \\
& \mp \sin \left((4 m+2) \theta_{i}\right) \sin 2 l \theta_{i},
\end{aligned}
$$

so that

$$
\begin{aligned}
\sum_{i=1}^{m} w_{i} T_{4 m+2 \pm 2 l}\left(x_{i}\right)= & \sum_{i=1}^{m} w_{i} \cos \left((4 m+2) \theta_{i}\right) \cos 2 l \theta_{i} \\
& \mp \sum_{i=1}^{m} w_{i} \sin \left((4 m+2) \theta_{i}\right) \sin 2 l \theta_{i} .
\end{aligned}
$$

Now 


$$
\begin{aligned}
\sum_{i=1}^{m} w_{i} \cos ((4 m+2) & \left.\theta_{i}\right) \cos 2 l \theta_{i} \\
& =\sum_{i=1}^{m} w_{i}\left(-1-\cos \left((4 i-1) \pi+t_{i} h_{i}\right) \frac{h_{i}^{2}}{2}\right) \cos 2 l \theta_{i} \\
& =-\sum_{i=1}^{m} w_{i} \cos 2 l \theta_{i}-\sum_{i=1}^{m} w_{i} \cos \left((4 i-1) \pi+t_{i} h_{i}\right) \frac{h_{i}^{2}}{2} \cos 2 l \theta_{i} \\
& =-\sum_{i=1}^{m} w_{i} T_{2 l}\left(x_{i}\right)+O\left(m^{-1}\right),
\end{aligned}
$$

since

$$
\sum_{i=1}^{n} w_{i}\left|\cos \left((4 i-1) \pi+t_{i} h_{i}\right) \frac{h_{i}^{2}}{2} \cos 2 l \theta_{i}\right| \leqq \sum_{i=1}^{m} w_{i} h_{i}^{2}=O\left(m^{-1}\right) .
$$

On the other hand,

$$
\sin \left((4 i-1) \pi+h_{i}\right)=\cos \left((4 i-1) \pi+u_{i} h_{i}\right) \cdot h_{i}
$$

where $0<u_{i}<1$, so that

$$
\left|\sum w_{i} \sin \left((4 m+2) \theta_{i}\right) \sin 2 l \theta_{i}\right| \leqq \sum w_{i}\left|h_{i}\right|,
$$

and we can show, as previously, that

$$
\sum_{i=1}^{[(m+1) / 2]} w_{i}\left|h_{i}\right| \leqq \frac{K}{m} \int_{\pi /(4 m+2)}^{\pi / 2} \cot x d x=O\left(\frac{\log m}{m}\right) .
$$

Hence, we have that

$$
\sum_{i=1}^{m} w_{i} T_{4 m+2 \pm 2 l}\left(x_{i}\right)=-\sum_{i=1}^{m} w_{i} T_{2 l}\left(x_{i}\right)+o\left(\frac{\log m}{m}\right), \quad l=1, \cdots, m .
$$

But

$$
\sum_{i=1}^{m} w_{i} T_{2 l}\left(x_{i}\right)=\int_{-1}^{1} T_{2 l}(x) d x=\frac{2}{1-4 l^{2}},
$$

for $l=1, \cdots, m-1$, since Gauss integration is exact for all polynomials of degree $<2 m$, while

$$
\sum_{i=1}^{m} w_{i} T_{2 m}\left(x_{i}\right)=\int_{-1}^{1} T_{2 m}(x) d x-E_{m}\left(T_{2 m}\right)=-\frac{\pi}{2}+O\left(m^{-1}\right) .
$$

Hence, we have finally

$$
\begin{aligned}
E_{m}\left(T_{4 m+2 \pm 2 l}\right) & =-\sum_{i=1}^{m} w_{i} T_{4 m+2 \pm 2 l}\left(x_{i}\right)+O\left(m^{-1}\right) \\
& =\frac{2}{1-4 l^{2}}+o\left(\frac{\log m}{m}\right), \quad l=1, \cdots, m-1, \\
& =-\frac{\pi}{2}+o\left(\frac{\log m}{m}\right), \quad l=m .
\end{aligned}
$$

Similarly, for $n=(2 m+1) j$, we have 


$$
\begin{aligned}
E\left(T_{(4 m+2) i \pm 2 l}\right) & =(-1)^{i} \frac{2}{4 l^{2}-1}+o\left(\frac{\log m}{m}\right), & & l=1, \cdots, m-1, \\
& =(-1)^{i} \frac{\pi}{2}+o\left(\frac{\log m}{m}\right), & & l=m .
\end{aligned}
$$

We can combine the results of Sections 3 and 4 in the following theorem:

THEOREM. As $m \rightarrow \infty$, the error in the $m$-point Gaussian integration of $T_{(4 m+2) i \pm 2 l}(x)$ approaches $(-1)^{i} \cdot 2 /\left(4 l^{2}-1\right), l=0,1, \cdots, m-1$, and $(-1)^{i} \cdot \pi / 2, l=m$.

Remark. Chawla [2] states that $\lim _{n \rightarrow \infty} E\left(T_{2 n+2 k+2}\right)=0$ for $k \geqq 1$, where $k$ may vary with $n$. This has been shown to be incorrect by our results. A possible source of error in [2] is the use of Eq. (10) there

$$
E_{G_{n}}\left(T_{k}\right) \simeq-\frac{i}{4} \int_{\sigma_{0}} \frac{\xi^{2 k+2}+\xi^{2}-\xi^{2 k}-1}{\xi^{2 n+k+3}} d \xi
$$

which is valid for a fixed $T_{k}(x)$, in the derivation of (11):

$$
\begin{aligned}
E_{G_{n}}\left(T_{k}\right) & \simeq \pi / 2 & & \text { if } k=2 n \\
& \simeq-\pi / 2 & & \text { if } k=2 n+2 \\
& \simeq 0 & & \text { if } k>2 n+2
\end{aligned}
$$

Department of Applied Mathematics

The Weizmann Institute of Science

Rehovot, Israel

Atomic Energy Research Establishment

Harwell, Didcot, Berkshire

England

1. M. Abramowitz \& I. A. Stegun (Editors), Handbook of Mathematical Functions with Formulas, Graphs, and Mathematical Tables, Nat. Bur. Standards Appl. Math. Series, 55, Superintendent of Documents, U.S. Government Printing Office, Washington, D.C., 1964. MR 29 \#4914.

2. M. M. Chawla, "Asymptotic estimates for the error of the Gauss-Legendre quadrature formula," Comput. J., v. 11, 1968/69, pp. 339-340. MR 38 \#5389.

3. D. Nicholson, P. Rabinowitz, N. Richter \& D. Zeilberger, "On the error in the numerical integration of Chebyshev polynomials, Math. Comp., v. 25, 1971, pp. 79-86.

4. P. Rabinowitz, "Error bounds in Gaussian integration of functions of low-order continuity," Math. Comp., v. 22, 1968, pp. 431-434. MR 37 \#2447.

5. P. RABINOWITZ, "Gaussian integration of functions with branch point singularities," Internat. J. Comput. Math., v. 2, 1970, pp. 297-306.

6. P. RaBINOWITZ, "Avoiding the singularity in numerical integration" (In preparation.)

7. P. RABINOWITZ \& N. RICHTER, "New error coefficients for estimating quadrature errors for analytic functions," Math. Comp., v. 24, 1970, pp. 561-570.

8. G. Szegö, Orthogonal Polynomials, rev. ed., Amer. Math. Soc. Colloq. Publ., vol. 23, Amer. Math. Soc., Providence, R.I., 1959. MR 21 \#5029. 